\title{
Construcción y validación del Cuestionario de Experiencias de Violencia en las Relaciones de Pareja y Familia en Estudiantes Universitarios*
}

Construction and validation of Experiences Questionnaire on Violence in Couple and Family Relations in University Students

Recibido: septiembre 8 de 2010 | Revisado: octubre 6 de 2010 | Aceptado: noviembre 3 de 2010

\author{
Ángel A. Villafañe Santiago** \\ MARÍA I. JIMÉNEZ CHAFEY \\ DAmaris De Jesús CARRASQuillo \\ ROBINSON A. VÁzQUEZ RAMOS \\ Universidad de Puerto Rico, San Juan, Puerto Rico
}

SICI: 2011-2777(201203)11:1<207:CYVCEV>2.0.CO;2-E

Para citar este artículo: Villafañe, A. A., Jiménez, M. I., De Jesús Carrasquillo, D. \& Vázquez, R. A. (2012). Construcción y validación del Cuestionario de Experiencias de Violencia en las Relaciones de Pareja y Familia en Estudiantes Universitarios. Universitas Psychologica, 11(1), 207-215.

* Artículo de investigación psicométrica.

** Recinto de Rio Piedras, P.O. Box 23137, San Juan, Puerto Rico 00931-3137. E-mails: angelpr39@ gmail.com, maria.jimenez16@upr.edu,damarisdj@ gmail.com, ravazquezramos@gmail.com

\section{RESUMEN}

Este estudio presenta el proceso de desarrollo del Cuestionario de Experiencias de Violencia en las Relaciones de Pareja y Familia en Estudiantes Universitarios, así como sus propiedades psicométricas y hallazgos a través de un estudio piloto. El diseño utilizado fue no experimental, transversal correlacional con una muestra por disponibilidad de 267 estudiantes. En la versión final, el instrumento consta de 41 reactivos y cuatro subescalas: Violencia de la Pareja hacia el Estudiante, Violencia del Estudiante hacia la Pareja, Violencia Observada entre los Padres y Violencia de los Padres hacia el Estudiante. La escala total y las subescalas obtuvieron índices de confiabilidad adecuados. En promedio, la muestra endosó diez experiencias de violencia en diversos contextos. Los hallazgos de este estudio aportan al conocimiento de la prevalencia de la violencia en múltiples contextos, viabilizando el diseño de intervenciones pertinentes en el manejo y prevención de la violencia en poblaciones universitarias.

Palabras clave autores:

Violencia, estudiantes universitarios, pareja, familia, cuestionario.

Palabras clave descriptores:

Psicología de familia, psicometría, pruebas, estudio transversal correlacional, estudio descriptivo.

\section{A B S T R A C T}

This study describes the process of developing the Experiences of Violence in Couple and Family Relationships in University Students Questionnaire, its psychometric properties and the results of the pilot study. The research design used for this study was a nonexperimental, transversal co relational design. The nonrandomized sample consisted of 267 students. The final version of the questionnaire consisted of 41 items and four sub-scales which measured experiences with violence in a relationship as an Aggressor and as a Victim, Observed between the Parents and in the Parent-child relationship as a victim. The total scale and the subscales obtained adequate reliability indexes. On average, the sample reported ten experiences with violence in different contexts. The results of this study contribute data on the prevalence of violence in college students' romantic and family relationships which in turn, provide valuable information for planning prevention and early intervention efforts with this population.

Key words authors:

Violence, couples, university students, family, questionnaire. 
Key words plus:

Family psychology, psychometric, tests, transversal co-relational research, descriptive study.

En las últimas décadas, se han realizado estudios cuyos resultados reflejan que la violencia en la familia tiene efectos negativos, tanto a nivel físico como emocional, en los niños que la presencian y que son víctimas de esto, y que esos efectos trascienden a la adultez (Margolin \& Gordis, 2000). La violencia es considerada un problema social y de salud a nivel mundial, presente en distintos estratos sociales y en diversos tipos de relaciones de pareja (Centers for Disease Control and Prevention, 2007). A esos efectos, se han realizado múltiples investigaciones en diferentes países, cuyos resultados sostienen que esta conducta es preocupante entre las parejas adolescentes y de jóvenes, e incluso su magnitud es superior a la de las parejas adultas (Jackson, Cram \& Seymour, 2000). En muchas ocasiones, la conducta violenta en las relaciones de pareja no es percibida como un problema ni por las víctimas ni por los agresores, debido a que algunas veces son interpretadas como situaciones momentáneas o aspectos que pueden mejorar o cambiarse, lo que podría conducir a mayor violencia.

Halpern, Young, Waller, Martin y Kupper (2004) realizaron estudios con parejas de adolescentes del mismo sexo, destacando que aproximadamente una cuarta parte de los/as participantes habían experimentado violencia en su relación. El National College Health Assessment realizó un estudio con una muestra de 11.408 universitarios norteamericanos (American College Health Association, 2006), revelando que $12.1 \%$ de los estudiantes había tenido relaciones emocionalmente abusivas, $1.9 \%$ relaciones físicamente abusivas y $1.5 \%$ relaciones sexualmente abusivas. Un estudio internacional realizado con población universitaria de 31 universidades de 16 países, reportó que un $29 \%$ del estudiantado había presentado conducta agresiva en sus relaciones (Straus, 2004). Por su parte, Kury, Obergfell-Fuchs y Woessner (2004) destacaron en su investigación que las jóvenes son objeto de más ataques violentos que las mujeres de mayor edad.
En los países de habla hispana también se han realizado varios estudios acerca de la violencia en las relaciones de noviazgo, en adolescentes y jóvenes adultos. Una investigación realizada en México por la Coordinación General de Investigación y Posgrado de la Universidad Autónoma de Sinaloa, bajo la dirección de Beatriz Eugenia Rodríguez Pérez (2007), encontró que 17.6 \% de las mujeres y $22.1 \%$ de los hombres entrevistados para el estudio, reconocieron haber experimentado violencia en su relación de pareja. Consistente con este hallazgo, Rivera-Rivera, Allen, Rodríguez-Ortega, ChávezAyala y Lazcano-Ponce (2006) reportaron que la prevalencia total de violencia durante el noviazgo en mujeres de escuelas públicas en México, fue de 28 \%. En Venezuela, Rodríguez Corvo (2007) realizó un estudio sobre los casos atendidos en terapia de pareja en la universidad y halló que el 62 \% se consultó por relaciones violentas en el noviazgo. En España, Muñoz-Rivas (2006), como investigadora principal, realizó un estudio con jóvenes universitarias donde se encontró que el 45.4 \% señaló que sus parejas les habían hecho algún comentario para molestarlas cuando mantenían una discusión, el $18.8 \%$ fueron insultadas y el $2.7 \%$ recibió amenazas de agresiones físicas. A grandes rasgos, se ve como la manifestación de la violencia se refleja en las relaciones de noviazgo. Esto podría conducir a los jóvenes a desarrollar conductas relacionadas al manejo de la sexualidad y comportamientos de respeto y equidad que afecten sus procesos a nivel social, personal y académico, y que redunden en un deterioro de valores, perturbándose todo el sistema familiar.

Guenard y Jiménez (1998) exploraron la experiencia de violencia sexual y física a través de una encuesta a 208 estudiantes universitarios, en Puerto Rico. El 6.8 \% indicó haber sido víctima de abuso sexual en el noviazgo y el 8.5 \% expresó que su pareja había ejercido presión para que sostuviera relaciones sexuales como una prueba de amor. En otro estudio, realizado por Alberdi y Rodríguez (2006) con 161 estudiantes de escuelas superiores, que tuvo como objetivo conocer las características específicas de la relación de noviazgo entre adolescentes asociadas a la conducta violenta, destacó 
que el $10 \%$ de los participantes indicó haber sido víctima de violencia en el noviazgo. Estos resultados son preocupantes, toda vez que podrían ser reflejo de la violencia recibida u observada durante el proceso de crianza. Según Sánchez Guzmán (2010), los niños testigos y/o víctimas de violencia intrafamiliar sufren consecuencias que arrastran por el resto de sus vidas, muchas de las cuales no son palpables hasta que no llegan a la adultez o cuando forman su propia familia.

Hay muchos estudios que sugieren que el haber experimentado o sido testigo de violencia en la familia de origen, aumenta el riesgo de ser víctima o agresor en la relación de pareja (Bush, Lundeberg \& Carlton, 2000; Ehrensaft et al., 2003; Kwong, Bartholomew, Henderson \& Trinke, 2003; Markowitz, 2001; Shook, Gerrity, Jurich \& Segrist, 2000; Whitfield, Anda, Dube \& Felitti, 2003). Bush et al. (2000) llevaron a cabo un estudio de meta-análisis que incluyó 39 investigaciones, para evaluar si la violencia en la familia tiene un efecto en la perpetuación o victimización en las relaciones de pareja en la adultez. Encontraron que el crecer en un hogar donde hubo violencia, estaba asociado significativamente con la violencia doméstica en la adultez.

Estudios sobre la transmisión intergeneracional de la violencia sugieren una asociación entre las experiencias de violencia en la familia de origen y la probabilidad tanto de recibir como de ejecutar algún tipo de abuso en la relación de pareja (Ehrensaft et al., 2003; Kwong, et al., 2003; Whitfield et al., 2003). Algunos investigadores, como Whitfield y sus colegas (2003), sugieren que el ser testigo de violencia doméstica duplica el riesgo de ser víctima del mismo tipo de violencia en la relación de pareja, mientras que otros investigadores, como Ehrensaft et al. (2003), indican que triplica dicho riesgo.

Tomando en consideración los argumentos discutidos, se hizo pertinente ponderar la posibilidad de estudiar la violencia en las relaciones de pareja en el ambiente universitario desde diferentes perspectivas. El objetivo principal fue crear un instrumento en el idioma español, que recopilara información sobre experiencias de violencia en jóvenes universitarios, en el contexto de sus relaciones de pareja y familia. Se pretendió también explorar si existía una relación entre violencia experimentada y observada durante la crianza y violencia en la relación de pareja.

\section{Método}

\section{Participantes}

El cuestionario fue administrado a una muestra por disponibilidad de 267 estudiantes de la Universidad de Puerto Rico, Recinto de Río Piedras. La mayoría de los participantes fueron mujeres (74\%). El $42 \%$ de la muestra estuvo constituida por estudiantes entre los 16 y 20 años de edad, $44 \%$ entre los 21 y 25 años y $14 \%$ eran mayores de 25 años de edad. El $83 \%$ de la muestra estaba cursando estudios a nivel subgraduado, mientras que el resto cursaba estudios graduados. De los participantes, 91 \% habían tenido alguna relación de pareja y $60 \%$ se encontraban en una relación de pareja al momento de completar el cuestionario. De aquellos que se encontraban en una relación de pareja, el $86 \%$ eran relaciones heterosexuales, $6 \%$ eran homosexuales y $0.7 \%$ lésbicas.

\section{Instrumento}

Para construir el Cuestionario de Experiencias de Violencia en las Relaciones de Pareja y Familia en Estudiantes Universitarios (CEV-RPF), se tomaron en consideración varias fuentes. En primer lugar, se consideraron los datos recopilados de un estudio exploratorio sobre las razones principales por las que los estudiantes universitarios solicitan servicios de consejería, en el Centro de Consejería donde trabajan los autores (Jiménez \& Villafañe, 2008). Entre los datos, sobresalieron que la relación de pareja estaba entre las tres principales dificultades y las situaciones de violencia doméstica fluctuaban entre 3 y 44 por año. Por otro lado, la experiencia clínica de los autores con esta población evidenció la necesidad de trabajar con estudiantes en relaciones de pareja maltratantes, e incluso se desarrolló un grupo de consejería para estudiantes mujeres que habían experimentado violencia en su relación de pareja (Villafañe, Jiménez, Capellas \& Collazo, 2009). 
Se realizó una revisión de literatura, para conocer algunas de las investigaciones realizadas y las distintas variables relacionadas con la violencia en pareja y en la familia (Bush et al., 2000; Fantuzzo et al., 1991; Margolin \& Gordis, 2000; Straus, 1979; Whitfield et al., 2003). También, se revisaron cuestionarios relacionados con la violencia en la pareja y la familia, y se utilizaron y adaptaron algunos reactivos en la construcción del instrumento (MuñozRivas, Andreu Rodríguez, Graña Gómez, O’Leary \& González, 2007; Straus, 1979; Straus, Hamby, Boney-McCoy \& Sugarman, 1996).

El instrumento fue sometido a un proceso de análisis de jueces. La versión final del cuestionario consta de 41 reactivos a los cuales se responde con "sí" o "no." Contiene cuatro subescalas dirigidas a explorar indicadores de violencia física y psicológica, en las siguientes áreas: 1) en la relación de pareja como víctima (Violencia de la Pareja hacia el Estudiante); 2) en la relación de pareja como agresor/a (Violencia del Estudiante hacia la Pareja); 3) violencia observada en la relación de los padres (Violencia Observada entre los Padres) y 4) de los padres hacia los hijos durante la crianza (Violencia de los Padres hacia el Estudiante). En cada subescala, los reactivos exploran eventos tanto de violencia física como de violencia psicológica. Entre los eventos de violencia física, se exploran acciones tales como jalones de pelo o golpes, destrucción de objetos y golpes a la pared. Por su parte, entre los eventos de violencia psicológica se exploran eventos tales como críticas, humillaciones, gritos, palabras insultantes, prohibición de salir, amenaza con objetos o armas, amenaza de quitarse la vida o quitársela a algún miembro de la familia, conducta controladora, conflictos por compartir con amigos y familiares y acusaciones de infidelidad sin motivo.

\section{Procedimiento}

Luego de obtener la autorización del Comité Institucional de Protección de Seres Humanos en la Investigación (CIPSHI) de la UPRRP, se procedió a llevar a cabo un estudio piloto. Se invitó a participar de la investigación a todos los estudiantes que habían asistido al Departamento de Consejería para el Desarrollo Estudiantil (DCODE), durante un periodo de tres meses. Se les entregó el cuestionario junto con una hoja informativa sobre los objetivos del estudio y su participación, de manera que pudieran dar su consentimiento libre y voluntariamente. Utilizando el mismo procedimiento, se invitó a participar a todos los estudiantes residentes de los dormitorios de la Universidad de Puerto Rico, Recinto de Río Piedras.

Se administró el Cuestionario de Experiencias de Violencia en las Relaciones de Pareja y Familia en Estudiantes Universitarios, junto con una hoja para recopilar datos sociodemográficos. Se recopiló información sobre edad, género, nivel de estudios e información relacionada con el núcleo familiar donde se desarrolló la mayor parte de la infancia. También se exploró si el participante había tenido alguna relación de pareja alguna vez y si tiene alguna en el presente, y el tipo de relación (heterosexual, homosexual, lésbica o bisexual). Los/as estudiantes tardaron aproximadamente 20 minutos en promedio, en completar los instrumentos.

El análisis estadístico de los datos se realizó utilizando el programa Statistical Package for Social Sciences (SPSS), Versión 17.0 para Windows.

\section{Diseño}

La presente investigación utiliza un diseño de estudio no experimental, transversal correlacional y corresponde a un estudio descriptivo mediante encuesta, según la clasificación de Montero y León. Los participantes no fueron sometidos a intervenciones, ni se manipularon variables independientes, por tratarse de un estudio instrumental, encaminado al desarrollo de una prueba y la descripción de sus propiedades psicométricas (Montero \& León, 2007).

\section{Resultados}

En la etapa de diseño y construcción del instrumento, se elaboraron 44 reactivos que se contestaban en una escala dicotómica (sí o no). Este tipo de escala cerrada de dos alternativas facilita el registro y codificación de las respuestas, y al mismo tiempo es 
de utilidad en investigaciones exploratorias, facilitando el proceso de identificación de la frecuencia con que los/as participantes reportan experiencias de violencia. El instrumento fue sometido a un proceso de análisis de jueces, en el que participaron tres profesionales de la conducta humana (dos consejeros y un trabajador social) con experiencia en el estudio de la violencia y en el campo de la metodología en investigación.

Se hicieron ajustes al instrumento tomando en consideración las recomendaciones de los jueces, orientadas principalmente a la reestructuración y eliminación de algunos reactivos y a cambios en la sintaxis de los reactivos. Se eliminaron dos reactivos cuyo contenido fue considerado como medición de las mismas experiencias de violencia psicológica. Luego de realizar un análisis de la correlación ítem-total corregida, en la versión final del instrumento se mantuvieron todos los 41 reactivos. Las correlaciones fluctuaron entre 0.23 y 0.53 . Se tomó la decisión de mantener los 4 reactivos que tuvieron correlaciones entre 0.23 y 0.29 , porque proveían información relevante basada en la experiencia clínica con esta población y porque, a su vez, permitían comparar el mismo tipo de experiencias en diferentes contextos (ej., amenazas con objeto o armas en la relación de pareja vs. observada entre los padres). Estos cuatro reactivos fueron: (1) En tus relaciones de pareja, ialguna vez amenazaste a tu pareja con quitarte la vida?, (2) En tus relaciones de pareja, ialguna vez amenazaste a tu pareja con un objeto o arma?, (3) En tus relaciones de pareja, ialguna vez amenazaste con quitarle la vida a tu pareja o quitársela a algún miembro de su familia? y (4) Entre tus padres o encargados/as, ialguna vez observaste a uno amenazar al otro con un objeto o arma?

La versión final consta de 41 reactivos divididos en cuatro subescalas. La escala total del CEVRPF obtuvo un índice de confiabilidad adecuado ( $\alpha=0.88$ ), de igual forma, el índice de confiabilidad de las subescalas también fue adecuado fluctuando entre 0.71 Y 0.82. Las primeras tres subescalas: Violencia de la Pareja hacia el Estudiante $(\alpha=0.82)$, Violencia del Estudiante hacia la Pareja $(\alpha=0.78)$ y Violencia Observada entre los Padres $(\alpha=0.82)$ contienen 11 reactivos cada una. La cuarta subescala, Violencia de los Padres hacia el Estudiante, está compuesta por 8 reactivos y obtuvo un alfa de 0.71 . Los promedios y desviaciones típicas de la escala total y las subescalas se encuentran en la Tabla 1. La escala total CEV-RPF obtuvo un promedio de $10.1(D E=7.3)$. Esto sugiere que la muestra endosó en promedio diez experiencias de violencia en la escala total en diversos contextos.

En la subescala de Violencia de la Pareja hacia el Estudiante el promedio fue de 2.6. Las experiencias de violencia que más reportaron los estudiantes fueron: haber recibido críticas o humillaciones por parte de su pareja (48\%), haber recibido gritos e insultos (47\%), que su pareja haya presentado conducta controladora ( $46 \%$ ) y que su pareja provocara conflictos porque el/la estudiante compartiera con amistades, familiares o compañeros/as de trabajo (41\%). En la subescala de Violencia del Estudiante hacia su Pareja el promedio fue de 2.3. En esta las experiencias de violencia que más reportaron los estudiantes fueron: haber gritado o insultado a su pareja (54\%), haber criticado o humillado a su pareja (39\%), haber destruido objetos o golpear la

\section{TABLA 1}

Promedios y desviaciones típicas de la escala total y de las subescalas de la CEV-RPF

\begin{tabular}{lccc}
\hline & N & Promedio & Desviación Estandar \\
\hline Escala total CEV-RPF & 246 & 10.11 & 7.29 \\
Subescala Violencia de la Pareja hacia el Estudiante & 260 & 2.68 & 2.52 \\
Subescala Violencia del Estudiante hacia la Pareja & 262 & 2.3 & 2.217 \\
Subescala Violencia Observada entre los Padres & 262 & 2.6 & 2.51 \\
Subescala Violencia de los Padres hacia el Estudiante & 267 & 2.18 & 2.138 \\
\hline
\end{tabular}

Fuente: elaboración propia. 
pared cuando estaba enojado/a (34\%) y presentar conducta controladora hacia su pareja (34\%).

En la subescala de Violencia Observada entre los Padres el promedio fue de 2.6. Las experiencias que más reportaron los estudiantes fueron: escuchar insultos (53\%), escuchar críticas y humillaciones entre ellos (46\%), observar conducta controladora (42\%) y observar alguno/a de ellos/as destruir objetos o golpear la pared cuando estaba enojado con el/la otro/a (30 \%). En la subescala de Violencia de los Padres hacia el Estudiante el promedio fue de 2.1. En esta subescala las experiencias de violencia que más reportaron los estudiantes fueron: conducta controladora (55\%); gritos, insultos o palabras hirientes (46\%); empujones, jalones de pelo o golpes físicos (42\%); criticas o humillaciones (32\%).

Se realizaron análisis correlacionales entre las subescalas, para explorar la posible relación entre violencia experimentada y observada durante la crianza y violencia en la relación de pareja. Los resultados sugieren una relación significativa entre todas las subescalas (Tabla 2). Las relaciones más fuertes fueron entre la violencia observada entre los padres y violencia de los padres hacia el estudiante, seguida por la violencia del estudiante hacia la pareja y de la pareja hacia el estudiante.

Es importante destacar que en total los incidentes de violencia psicológica que fueron reportados con mayor frecuencia por los participantes fueron: gritar y decir palabras insultantes (54\%), criticar y humillar (39\%) y presentar conducta controladora (34\%). Por otro lado, los incidentes de violencia física que se reportaron con mayor frecuencia fueron: destruir objetos o golpear a la pared (34\%) y jalar el pelo, empujar o pegar (16\%).

\section{Discusión}

El CEV-RPF fue creado para medir experiencias de violencia física y psicológica en el contexto de las relaciones de pareja y familia de estudiantes universitarios, utilizando como base la literatura sobre violencia en la pareja y la familia, la revisión de cuestionarios existentes, la experiencia clínica y el proceso de análisis de jueces. El instrumento mostró tener validez de contenido, debido al proceso de construcción que fue cónsono con la revisión de literatura y el análisis de expertos en el tema. Presentó también una confiabilidad alta reflejada en el alfa obtenida para la escala total y para cada una de las subescalas en el estudio piloto. Esto implica que el instrumento mide las variables bajo estudio y que están debidamente representadas en los reactivos, ya que los participantes del estudio endosaron en promedio la prevalencia de diez experiencias de violencia en la escala total.

Los resultados de la administración del instrumento son consistentes con otros estudios sobre la prevalencia de las experiencias de violencia en las relaciones de noviazgo en los jóvenes, así como la relación entre haber experimentado violencia en la familia durante la crianza y la violencia en la pareja en la adultez (Bush et al., 2000). A su vez, estos resultados ofrecen apoyo a las teorías de transmisión de violencia transgeneracional, que sostienen que la violencia familiar es una conducta aprendida que pasa de generación en generación. Esto significa que la exposición de menores a la violencia tiene como repercusión social el aprendizaje que hacen los menores de las conductas violentas dentro de su hogar (García de la Torre, 2006). A esos efectos,

TABLA 2

Relaciones entre las subescalas de la CEV-RPF

\begin{tabular}{lccc}
\hline \multicolumn{1}{c}{ Subescalas de Violencia } & Pareja hacia el Estudiante & Estudiante hacia la Pareja & Observada entre los Padres \\
\hline Estudiante hacia la Pareja & $0.546^{* *}$ & & \\
Observada entre los Padres & $0.209^{* *}$ & $0.197^{* *}$ & \\
Los Padres hacia el Estudiante & $0.169^{* *}$ & $0.234^{*}$ & $0.635^{* *}$ \\
\hline
\end{tabular}

$* * p>0.01$

Fuente: elaboración propia. 
esta investigación aporta al entendimiento del proceso de violencia intrafamiliar y sus repercusiones en el ambiente universitario, debido a que son esos hijos impactados por la violencia los que pasan por las aulas universitarias y a su vez van expresando manifestaciones de violencia.

Este instrumento facilita la recopilación de datos sobre las experiencias de violencia más comunes y permite la correlación entre las mismas en diferentes contextos, y a su vez ofrece apoyo adicional a la literatura existente sobre la relación entre violencia en la familia y violencia en las relaciones de pareja en la adultez. Conocer la prevalencia y la relación entre las variables bajo estudio, sirve para diseñar y planificar intervenciones en el escenario universitario, dirigidas al manejo y prevención de la violencia entre parejas. Permite tener un panorama más amplio sobre el potencial de riesgo del estudiante de ser víctima o agresor, y también contribuye a conocer el tema de la violencia entre parejas universitarias, ya que la literatura existente hace mayor énfasis en la violencia doméstica en parejas casadas o que conviven (Lewis \& Fremouw, 2001).

La muestra de este estudio piloto reportó en promedio diez experiencias de violencia en diferentes contextos, lo que sugiere que casi una cuarta parte de los participantes había experimentado violencia. En las relaciones de pareja, la violencia psicológica fue la más frecuente con porcentajes entre 34 y $48 \%$ para diferentes formas de violencia psicológica. Estos datos preliminares señalan la necesidad de realizar intervenciones dirigidas a desarrollar destrezas de manejo de conflictos y comunicación asertiva en las relaciones de pareja con esta población. Una segunda etapa del estudio se está llevando a cabo para continuar estudiando las propiedades psicométricas del CEV-RPF, para proveer un instrumento que pueda ser utilizado en otros escenarios universitarios. A esos efectos, se ha ampliado la muestra del estudio y se incluirán otros recintos (dependencias) de la Universidad de Puerto Rico. Los resultados que surjan con esta muestra más amplia contribuirán a una mayor información sobre la prevalencia de estas experiencias, que permita el desarrollo de intervenciones más específicas dirigidas a esta población.
$\mathrm{Al}$ ser un instrumento de autoinforme, el CEVRPF podría presentar una limitación del procedimiento, ya que las contestaciones pueden variar según la interpretación que cada participante le dé a algunos conceptos, como por ejemplo el de conducta controladora. Por otro lado, ampliar la muestra del estudio y refinar el instrumento podrían enriquecer el conocimiento sobre el tema y arrojar evidencia para realizar futuras investigaciones.

\section{Referencias}

Alberdi, M. \& Rodríguez, S. (2006). La violencia en el noviazgo entre adolescentes de escuela superior. Tesis inédita, Escuela Graduada de Trabajo Social de la Universidad de Puerto Rico, Recinto de Río Piedras, San Juan.

American College Health Association. (2007). American College Health Association, National College Health Assessment Spring 2006 Reference Group Data Report. Journal of American College Health, 55(4), 195-206.

Bush, A. L., Lundeberg, K. \& Carlton, R. P. (2000). The intergenerational transmission of spouse abuse: A meta-analysis. Journal of Marriage and Family, 62, 640-654.

Centers for Disease Control and Prevention, National Center for Injury Prevention and Control. (2007). Youth Violence: Fact Sheet. Department of Health and Human Services. Recuperado el 7 de septiembre del 2010, de http://www.cdc.gov/ncipc/ factsheets/yvfacts.htm

Ehrensaft, M. K., Cohen, P., Brown, J., Smailes, E., Chen, H. \& Johnson, J. G. (2003). Intergenerational transmission of partner violence. Journal of Consulting and Clinical Psychology, 71, 741-753.

Fantuzzo, J. W., DePaola, L. M., Lambert, L., Martino, T., Anderson, G. \& Sutton, S. (1991). Effects of interparental violence on the psychological adjustment and competencies of young children. Journal of Consulting and Clinical Psychology, 59(2), 258-265.

García de la Torre, S. (2006). La violencia de género como causa de maltrato infantil. Cuadernos de Medicina Forense, 12(43-44), 149-164. 
Guenard, E. \& Jiménez, M. (1998). Jóvenes victima de violencia en la relación de pareja: percepción de la violencia, patrones de crianza, escolarización y expectativas de roles en la relación de pareja en los jóvenes estudiantes de la Universidad de Puerto Rico, Recinto de Río Piedras, Humacao, Cayey y Utuado. Tesis inédita, Universidad de Puerto Rico, Recinto de Río Piedras, San Juan.

Halpern, C. T., Young, M. L., Waller, M. W., Martin, S. L. \& Kupper, L. L. (2004). Prevalence of partner violence in same-sex romantic and sexual relationships in a national sample of adolescents. Journal of Adolescent Health, 35, 124-131.

Jackson, S. M., Cram, F. \& Seymour, F. W. (2000). Violence and sexual coercion in high school students'dating relationships. Journal of Family Violence, 15, 23-36.

Jiménez Chafey, M. I. \& Villafañe Santiago, A. A. (2008). Necesidades psicosociales de los estudiantes que han recibido servicios del Departamento de Consejería para el Desarrollo Estudiantil (DCO. DE) de la Universidad de Puerto Rico, Recinto de Río Piedras. Cuaderno de Investigación en la Educación, 23, 193-209.

Kury, H., Obergfell-Fuchs, J. \& Woessner, G. (2004). The extent of family violence in Europe: A comparison of National Surveys. Violence against Women, 10, 749-769.

Kwong, M. J., Bartholomew, K., Henderson, A. J. Z. \& Trinke, S. J. (2003). The intergenerational transmission of relationship violence. Journal of Family Psychology, 17, 288-301.

Lewis, S. \& Fremouw, W. (2001). Dating violence: A critical review of the literature. Clinical Psychology Review, 21, 105-127.

Margolin, G. \& Gordis, E. B. (2000). The effects of family and community violence on children. Annual Review of Psychology, 51, 445-479.

Markowitz, F. (2001). Attitudes and family violence: Linking intergenerational and cultural theories. Journal of Family Violence, 16, 205-218.

Montero, I. \& León, O. G. (2007). A guide for naming research studies in Psychology. International Journal of Clinical and Health Psychology, 7, 847-862.

Muñoz-Rivas, M. (2006). Violencia contra la mujer en las relaciones de noviazgo: causas, naturaleza y con- secuencias (Proyecto NIPO: 207-07-107-XISBN: 978-84-690-9058-9. Ref: 701-50/03). Madrid: Universidad Autónoma de Madrid.

Muñoz-Rivas, M., Andreu Rodríguez, J., Graña Gómez, J., O’Leary, D. \& González, M. (2007). Validación de la versión modificada de la Conflicts Tactics Scale (M-CTS) en población juvenil española. Psichotema, 19, 693-698.

Rivera-Rivera, L., Allen, B., Rodríguez-Ortega, G., Chávez-Ayala, R. \& Lazcano-Ponce E. (2006). Violencia durante el noviazgo, depresión y conductas de riesgo en estudiantes femeninas. Cuernavaca, México: Instituto Nacional de Salud Pública.

Rodríguez Corvo, D. (2007). Noviazgo violento: Caso ULA. Universidad de los Andes, Ciudad de Mérida, Venezuela. Recuperado el 7 de septiembre del 2010, de http://www.uacj.mx/UniversidadSaludable/memorias/documentos/PDF\%20Resumenes/ LA\%20EXPERIENCIA\%20DESDE\%20LA\%20 \%20DIRECCI\%C3\%93N\%20DE\%20ASUNTOS\%20ESTUDIANTILES,\%20DAES.pdf

Rodríguez Pérez, B. E. (Dir.). (2007). Proyecto de investigación:"Violencia en el noviazgo. Relaciones dolorosas que trascienden". Recuperado el 9 de septiembre del 2010, de http://interpol.uasnet.mx/ serviciosocial/

memorias/MSS-098-2007.pdf

Sánchez Guzmán, A. (2010). Soy testigo y/o víctima de violencia intrafamiliar. Centro Vida y Familia Ana Simó. Disponible en http://www.centrovidayfamilia.com

Shook, N. J., Gerrity, D. A., Jurich, J. \& Segrist, A. E. (2000). Courtship violence among college students: A comparison of verbally and physically abusive couples. Journal of Family Violence, 15, 1-22.

Straus, M. (1979). Measuring intrafamily conflict and violence: The Conflict Tactics (CT) Scales. Journal of Marriage and the Family, 41, 75-88.

Straus, M. (2004). Cross-Cultural reliability and validity of the Revised Conflict Tactics Scales: A study of university student dating couples in 17 nations. Cross-Cultural Research, 38, 407-432.

Straus, M., Hamby, S., Boney-McCoy, S. \& Sugarman, D. (1996). The revised Conflict Tactics Scales (CTS2): Development and preliminary psychometric data. Journal of Family Issues, 17, 283-316. 
Villafañe Santiago, A., Jiménez Chafey, M., Capellas, M. \& Collazo, S. (2009). Un modelo de consejería grupal para estudiantes impactadas por la violencia en su relación de noviazgo. Revista de Ciencias Sociales, 4(126), 45-58.
Whitfield, C. L., Anda, R. F., Dube, S. R. \& Felitti, V. J. (2003). Violent childhood experiences and the risk of intimate partner violence. Journal of Interpersonal Violence, 18, 166-185. 
